\title{
Collaborative Systems Thinking Research: Exploring systems thinking within teams
}

\author{
Caroline T. Lamb \\ cmtwomey@mit.edu \\ (617) 308-0954 \\ Donna H. Rhodes \\ rhodes@mit.edu \\ (617) 324-0473 \\ Massachusetts Institute of Technology \\ 77 Massachusetts Avenue NE20-388 \\ Cambridge, MA 02139
}

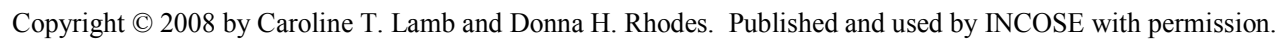

\begin{abstract}
This paper describes ongoing research that seeks to develop an empirical basis for collaborative systems thinking, defined as "an emergent behavior of teams resulting from the interactions of team members and utilizing a variety of thinking styles, design processes, tools, and communication media to consider system attributes, interrelationships, context and dynamics towards executing systems design". This type of thinking is critically important to addressing engineering systems challenges, and the research seeks to inform and enable effective systems engineering practice in contemporary engineering enterprises. Focusing on the aerospace domain, collaborative systems thinking is examined through the alignment of enterprise culture and standard technical processes. This paper draws on a variety of literature to compose a definition of collaborative systems thinking and propose a research agenda going forward.
\end{abstract}

\section{Motivation}

The engineering of complex systems involves very large and diverse teams working together to address complex challenges (Saunders et al 2005). These teams are increasingly diverse, requiring sophisticated problem solving skills and approaches. In many cases teams are geographically dispersed, introducing logistical challenges and different cultural influences. . These factors present challenges for effective systems engineering, particularly on large scale endeavors, where collaborative systems thinking is especially critical to achieve a successful outcome.

The ability to effectively address systems engineering opportunities is also challenged by the decline and erosion of the engineering workforce. Several studies cite an erosion of engineering competency, particularly in government and aerospace/defense industry. Further the attrition rate due to an aging workforce is significant. These challenges were described an earlier paper (Lamb and Rhodes 2007).

The study of Collaborative Systems Thinking (CST) is a very broad area of inquiry, crossing many disciplines and bodies of knowledge. In order to scope this exploratory research, the authors focus on the alignment of enterprise culture and standardized process as one means to gather empirical knowledge and position this within a collection of past, current, and future research topics that will, in combination, provide comprehensive new knowledge to improve systems engineering practice. 


\section{What is Systems Thinking?}

Systems thinking is a thinking style engineers engage when designing complex systems. Systems thinking enables better handling of complexity, better design decisions, and the consideration of dynamic interfaces and interrelationships within a system (Davidz 2006).

Systems thinking is an age old concept. Eastern philosophies emphasize the importance of wholes and the multitude of interconnections that exist in nature. In the modern world, systems thinking has its roots in the development of systems theory in the 1930 's. Systems dynamics, systems science, and systems engineering all have their own definitions for systems thinking. The commonalities between these definitions include an emphasis on wholes, system-level issues, and some derived ability to judge and choose between alternatives based on their system-wide impact. For a thorough discussion of the different definitions of systems thinking, see (Davidz 2006). The following is a brief treatment of the topic.

Generic definitions of systems thinking vary, defining the skill from the use of one's abilities to apply sound reasoning in a given situation (Dorner 1996), to the application of multiple different styles of thinking. One such generic definition is from Russell Ackoff, who defines systems thinking as a systemic mode of thinking based on holistic as opposed to reductionistic methods (Ackoff 2004). Within Ackoff's framework, reductionistic thinking is that which starts with the parts and from there derives the properties of the whole. Holistic thinking, by contrast, begins with the whole, from which the properties of the parts are derived (Ackoff 2004). This definition is predicated on the principle that systems are greater than the sum of their parts, and cannot therefore be completely described by their parts.

Systems thinking definitions derived from systems science include and build upon the components of the generic definitions, emphasizing the roles of holism, interactions, and dynamics. Definitions based in systems science are typified by an emphasis on identifying patterns of behavior and representing these patterns through cause-effect relations (Richmond 1993). To support exploration of these cause-effect relationships, systems thinking is supported by "a body of knowledge and tools developed over the past 50 years to make full patterns clearer and to help us see how to change them effectively "(Senge 2006). One such tool is systems thinking diagrams, a method of visualizing system behavior through a series of feedback loops, stocks (accumulations), and flows (actions that influence stocks) (Senge 2006). Figure 1 is a summary of common systems thinking definitions and their recurring themes.

The definitions presented in Figure 1 come primarily from the systems science community, but all focus on interactions and connections. These align with the concept that systems thinking within complex systems is a departure from linear thinking, which is rooted in simple cause and effect relationships (Volger 2002).

While these definitions suit the basic applications of systems thinking within the engineering community, the consideration of the systems as a whole and elucidating patterns of behavior and interactions, engineers' goals are primarily to manipulate technology, manage systems with ill-understood cause and effect relationships and to apply systems thinking before the system is realized, thus limiting their ability to learn through observing the system. As such, the engineering definitions of systems thinking place a greater role on interactions and interfaces because these contribute to emergence. 
A framework for systems with four basic ideas: emergence, hierarchy, communication and control. Human activity concerns all four elements. Natural and designed systems are dominated by emergence.

A method of placing the systems in its context and observing its role within the whole.

(Gharajedaghi 1999)

A skill to see the world as a complex system and understanding its interconnectedness.

A skill of thinking in terms of holism rather than reductionism.

(Ackoff 2004)

A method and framework for describing and understanding the interrelationships and forces that shape system behavior.
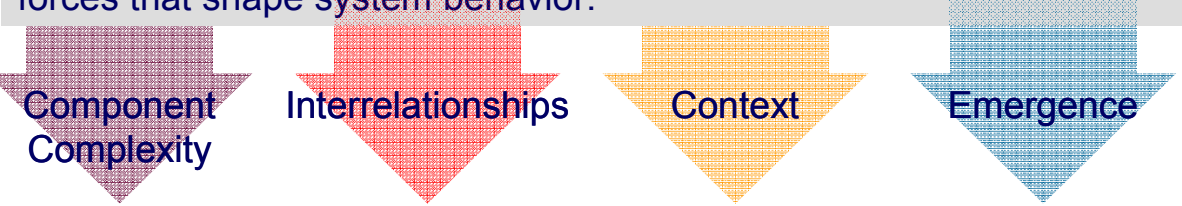

(Senge 2006)

Figure 1. Varying systems thinking definition share the recurring themes of complexity, interrelationships, context, emergence, and wholes.

To determine how engineers define systems thinking, Dr. Heidi Davidz conducted over 200 interviews with practicing systems engineers towards the development of an engineering definition of systems thinking. While her research indicates there is divergence within the engineering community as to what specifically systems thinking is, she also found convergence around key properties. From the data collected, the following definition of systems thinking was developed.

Systems thinking is "utilizing modal elements to consider the componential, relational, contextual, and dynamic elements of the system of interest" (Davidz 2006)

In other words, effective engineers use a variety of tools, methods, thinking styles, models and processes to enable consideration of the context, interrelationships, and dynamics of a system and its elements.

The skills and benefits of systems thinking are associated with problem solving (Jansma and Jones 2006). To this end, lists of systems thinking skills have been developed to help in understanding the role of systems thinking in system design. These skills include the ability to understand dynamic systems behavior and to identify patterns resulting from interactions (Sweeney and Sterman 2000; Richmond 1993). In modern engineering, social skills are just as important as technical skills. Systems thinking, with its emphasis on social and technical interactions and influences enables engineers to better mobilize, organize and coordinate resources (human, financial and physical) towards the completion of systems design (Beder 1999). When dealing with individual engineers, experiential learning, individual traits and a supportive environment are the greatest enablers (and barriers) towards the development of systems thinking skills (Davdiz 2006). 


\section{Thinking as a Team Construct}

. According to the Merriam-Webster dictionary, to think is to form an opinion, or an intention to act. Within an engineering context, thinking is purposeful, reasoned and goaldirected action towards the solving of problems (Lynn et al 2005). The elements of thinking in this context are decision making, problem exploration (creativity), the judging of alternatives, and ultimately the selection of a solution (Lynn et al 2005). The process has as inputs a need or problem and recalled knowledge.

In a team setting, the elements of thinking are manifest in team interactions, making the extension of thinking to the team level a logical step. Within teams, social interactions stimulate information recall, information interpretation, and ultimately, the judgment and selection between alternatives (Lynn et al 2005).

Because teams can leverage the knowledge, experience, and interpretation of multiple people, teams are deemed better at making decisions, especially in safety critical situations. However, team skills are more difficult to develop as they must be practiced as a team (Senge 2006).

Team thinking is like having parallel processors: it only works with communication between the processors (Entin et al 2004). Team thinking emerges from the intersection of individual team members' thinking, their behaviors and team processes (Cooke et al 2004). Like with systems, team thinking is greater than the sum of the individuals' thoughts (Cooke et al 2004). Throughout the process of problem solving, teams use communication to stimulate their thinking and handle uncertainty inherent in design (Lynn et al 2005). Brainstorming, team norms, and processes enable this communication (Lynn et al 2005).

While team thinking is a recognized construct, there are few recognized measures (Lerch et al 2004). Team mental models, often touted for measuring team knowledge,

are ineffectual at measuring team thinking because team mental models emphasize the homogeneity in team knowledge, whereas the strength in team thinking comes from the heterogeneity of team member knowledge (Cooke et al 2004). Good measure of team thinking must address its holism as well as its emergent and dynamic nature (Cooke et al 2004). The following are a few proposed measures of team thinking.

1) Anticipation Ratio The anticipation ratio is a measure of how well team members anticipate others' needs for information and preemptively provide the necessary information, thus making communications more efficient. (Entin et al 2004)

2) Mutual Awareness

Mutual awareness within a team is achieved when team members are aware of each other's activities within the design context. (Entin et al 2004)

3) Situational Awareness

Teams that better understand their situation and the task at hand, perform better. Situational awareness measures a team's collective awareness of its environment, tools, and procedures. (Cooke et al 2004) 


\section{Design Thinking within Teams}

One area of team thinking that informs CST research is team-based design thinking. There exists a large body of research on the ways in which team execute design, focusing on the role of communication, process, and team behavior in enabling successful design.

Briefly, the design process has five basic elements: analysis of the need or problem (exploration of problem space), generating ideas to address this need (the creative process), evaluation of those alternatives (comparison and selection), initial design, and final detailed design (Thompson and Lordan 1999). Within engineering, this process is systematic and developed by designers to aid in the design of systems or processes that satisfy an end user's needs within a set of constraints (Dym et al 2005).

During the design process, several types of thinking are engaged. Roughly, these thinking types can be categorized as either divergent or convergent. Divergent thinking operates in the concept domain, encapsulating the steps of generation and exploration (Dym et al 2005). Convergent thinking operates in the knowledge domain and includes comparison and selection (Stempfle and Baudke-Schaub 2002).

While the creative process requires both divergent thinking to explore the problem space and convergent thinking to act upon that exploration, the majority of engineers express a preference for convergent thinking (Durling 2003; Stempfle and Bauke-Schaub 2002). This rush towards convergent thinking is a natural thinking mode engaging heuristics to reduce complex situations into manageable pieces and enable quick decisions despite uncertain information (Gigerense 1999), the very context of engineering, even thought purely convergent thinking can lead to lower quality outcomes. This pattern of behavior is an example of how individual traits influence thinking style.

Effective design thinking includes both convergent and divergent components, enabling for the exploration of the problem space and critical analysis of the solutions space (Dym et al 2005). Characteristics of effective design thinking include the ability to tolerate uncertainty, keep sight of the big picture, make decisions despite ambiguity, think and take action as a team, and to communicate using the multiple languages of design (Dym et al 2005). The references to big picture thinking and tolerating uncertainty draw clear parallels between design thinking and systems thinking. However, design thinking specifically references the ability to think as a team, making it a logical bridge between systems thinking and collaborative systems thinking. As such, the enablers, barriers and traits of design thinking are extremely pertinent to research into collaborative systems thinking.

As Einstein said, imagination is more important than knowledge. Consistent with this belief, Einstein advocated for an emphasis on capability building rather than information gathering (Stephens 2003). Being creative is, after all a skill or capability-a way of thinking-rather than a knowledge base. Exploration and concept generation are among the first steps in the design process, and both require divergent thinking and creativity. A creative environment facilitates design by enabling teams to break with previous patterns of thought to explore new regions of the goal space (Thompson and Lordan1999). Yet, engineers often have a "them vs. us" attitude that inhibits their own creative environment (Wesenberg 1989).

Observations of teams solving design problems shed some light on effective patterns and maintaining a creative environment for complex systems design. In one such study, teams spent on average two-thirds of their time addressing the content of the design 
problem and one-third addressing the process by which to address the design task (Stempfle and Badke-Schaub 2002). Most time was spent on analysis, or examining the elements of the design space and their interrelationships. Consequently, very little time was spent generating solutions based upon this analysis. Rather, following human tendencies towards satisficing, some workable solution was quickly passed through analysis, and only after it failed was the goal space further explored (Stempfle and Badke-Schaub 2002).

Out of these observations, two natural modes of design were identified. The first process most resembles the natural thinking of an engineer. The process relies heavily on convergent thinking, narrowing the design space early by failing to first ask critical questions. Emphasis was placed on team harmony and maintaining the status quo. While this first process is quick, it does not handle complexity well because of the rush to evaluate the first design proposed instead of engaging in early and detailed analysis of the problem (Stempfle and Badke-Schaub 2002). The second process more resembles the processes defined by normative design theory. It is rational and focuses on problem analysis. The second process is more time consuming and requires more team interaction. However, because more time is spent up from on early analysis, the quality of designs is better and the process can better deal with complexity (Stempfle and BadkeSchaub 2002).

The difference between the first and second process is the ability or willingness of the team to engage in early critical questioning, thus prolonging the analysis stage of design. This willingness is linked to organizational culture and team sub-culture. Early critical analysis is most likely in heterogeneous groups with a culture that is receptive to questioning, and therefore can support divergent thinking styles (Stempfle and BadkeSchaub 2002).

\section{Collaborative Systems Thinking}

Systems thinking incorporates traits that at times appear contradictory. Systems thinkers should be detail oriented and methodical, yet also be creative and think "out of the box." Davidz identified these seeming contradictions over the course of her research (Davidz 2006). While it is difficult to conceive of one individual encompassing all these traits, a team with many members can more easily express this diverse set of traits.

This conclusion is supported by research that shows teams with heterogeneous composition outperform homogeneous teams. Mixes in thinking style preferences (Culp and Smith 2001) and knowledge (Mohammed and Dumville 2001) are both critical.

Successful team design thinking engages a variety of thinking styles, a learning environment and a variety of means to communicate (Dym et al 2005). These teams show curiosity towards the problem space, generate large numbers of alternative solutions and then engage in evaluation (Lynn et al 2005), following an analytic design process (Stempfle and Badke-Schaub 2002). The analytic design process proceeds from idea generation to problem analysis before transitioning to evaluation and onto detail design. By engaging in analysis before evaluation, these teams spend more time engaging in divergent thinking. As stated earlier, cycling between divergent and convergent thinking is an enabler for team success (Dym et al 2005). The willingness to ask questions, and thus engage in divergent thinking, is an indicator of a culture that supports learning. Finally, for a team to effectively communicate, multiple languages are needed. The 
languages of design include text and speech, graphics (e.g. sketching and part drawings), shape grammars, executable mathematical models, and numbers (Dym et al 2005). Communicating enables teams to keep a clear mission (Waszak et al 1998). Interestingly, team mental models, or shared representations of tasks, equipment and working relationships, have not been shown to positively impact team performance (Mohammed and Dumville 2001).

Taking these inputs, the following definition for collaborative systems thinking is derived and proposed.

Collaborative systems thinking is an emergent behavior of teams resulting

from the interactions of team members and utilizing a variety of thinking styles, design processes, tools, and communication media to consider systems attributes, interrelationships, context and dynamics towards executing systems design.

This definition will be used to execute field research, and will be updated as warranted by feedback from data collected.

\section{Research Approach}

This research will focus on the role of organizational culture and standard process usage in promoting CST. Exploratory case studies, utilizing a combination of surveys, interviews, and primary documentation are used to explore relevant constructs towards identifying which aspects of culture and process impact CST.

Systems engineering is a discipline born out of practice. As such the theories governing systems engineering must be grounded in that practice. Exploratory research methods offer a means to collate observations of practice into new theory. Whereas traditional deductive research starts with a hypothesis and then seeks evidence to prove or disprove the hypothesis, exploratory research starts with an interesting question or area of inquiry and ends with a set of hypotheses that form the basis for new theory (Glaser and Strauss 1967). This is known as grounded theory research.

Recent and ongoing systems engineering research at MIT has made use of grounded theory. This research will take advantage of the methods and lessons learned from those studies. The use of grounded theory research completes the research continuum. Whereas most engineering research tests existing hypotheses, exploratory and grounded theory research are theory building, thus developing new hypotheses to test.

For CST research, teams are the fundamental unit of analysis. Teams selected will represent a variety of aerospace product. Both space systems and aviation systems, large volume systems and one-off systems are considered. No universally accepted measure exists for evaluating an individual's systems thinking capability. The same is true for team-level systems thinking. As such, outside indicators (e.g. manager interviews) and internal indicators (e.g. team self-reporting) will be used to judge a team's level of collaborative systems thinking. Methods borrowed from social science will facilitate data collation and analysis.

\section{Expanded Research Agenda and Future Directions}

The research area of systems thinking draws from a variety of fields, with recent and ongoing work at MIT as well as many other universities. Systems thinking will be an increasing necessary and important skill for engineering leaders in this century (Rhodes 
and Hastings 2004). The research requires not only knowledge of the engineering field, but also an understanding of cognition, psychology, organizational behavior education, teamwork studies, and other fields (for example, Gardner, Pinker, Schein, Nadler, Senge, Hackman, Belbin).

The authors have defined a research agenda for collaborative systems thinking within an overall research portfolio topic of systems engineering in the enterprise. The research seeks to impact the effectiveness of systems engineering in modern enterprises, through development of new empirical based knowledge related to systems thinking and practice. At this point in time, the studies are largely exploratory in nature, with research methods rooted in grounded theory (Glaser and Strauss 1967). However, the research is resulting in many findings that can begin to be applied in practice, as well as shape additional studies to build this base of knowledge. There are many topics to be explored in context of this overall research theme; eight areas of interest to our further research are:

1. Empirical Studies of Systems Thinking in Individuals and Teams: The MIT research group has performed several empirical studies on systems thinking in individual engineers. In the largest of the past studies, (Davidz 2006) identified enablers, barriers and precursors to the development of systems thinking in individual systems engineers in the aerospace domain. Additional work is needed on individual systems thinking to further explore and validate findings across domains. This work informs the research described in this paper on enablers and barriers for collaborative systems thinking.

2. Stages of Teams/Lifespan of CST Team: Previous research has identified stages for the formation and lifespan of teams, for example, teams go through phases of forming, norming, etc. Research is needed to examine this defacto standard in context of collaborative systems thinking teams. How does collaborative systems thinking emerge in context of these stages? Is there an optimal lifespan for a CST team? Are there points when the team exhibits stronger or weaker CST, and any interventions that could encourage CST performance?

3. Impact of Enterprise Capability Maturity on CST: A question of interest is whether a higher capability maturity enterprise is any more likely to exhibit CST behavior than a lower maturity one. Alternately, is this high level of maturity an inhibitor to CST, wherein a start-up company more likely to exhibit CST? The interrelationship of capability maturity and individual and collaborative systems thinking should be explored.

4. Impact on Knowledge Transfer: A similar question worthy of research is the relationship to knowledge transfer within and across teams. Based on early observations it is hypothesized that CST teams are more proactive in knowledge transfer and mentoring, both within the team and external to the team. This needs to be examined more rigorously to determine if there is empirical evidence for this observation, and what impact the enterprise context has on this as well.

5. CST in the Distributed Enterprise: An exploratory study by (Utter 2007) examined collaborative distributed systems engineering in the aerospace industry, identifying various technical and social success factors, enablers and barriers. Further research is needed to look at collaborative distributed systems engineering across various types of enterprises. Some of the questions to be explored include: 
are there unique factors involved in developing effective CST teams when they are geographically dispersed? What social and technical factors can enable or inhibit the development of CST? Is CST more or less likely to occur in distributed teams?

6. CST in Context of Domain and Type. CST is a very broad topic, and there are obvious variations in accordance with the types of enterprises and work activities, in addition to domains. Research to investigate CST in content of environment and application domain is needed to inform strategies and practices for application to suit the context. .

7. Integration of CST in Competency Models. There is significant work in developing competency models in support of development of a systems workforce, and it can be noted that collaborative systems thinking is increasingly required. Competency models are used at the individual level. Research will be beneficial to determine if a team competency model may be needed and/or if the individual competency models need to be enhanced to develop skills leading to CST behaviors.

8. ROI of CST: The authors believe that enterprises will need to invest in workforce development, incentives, and enablers to fully achieve the benefits of CST. Research is needed to determine what the appropriate types and levels of investment are needed, and what the return on investment is for the organization.

These eight topics are described to show examples of possible topics related to collaborative systems thinking. There are many other topics to be explored in the interests of improving the effectiveness of systems engineering practice.

\section{Conclusions}

There is an urgent need to develop and utilize collaborative systems thinking to address engineering challenges in the modern world. To date, there has been little research related to the development of systems thinking in engineers as individuals, and particularly for collaborative engineering teams. The research project described in this paper is exploratory in nature, and seeks to provide new insights the enablers and barriers for collaborative systems thinking. Further, the research is expected to result in new understanding regarding the applicability of selected methods for performing systems thinking research. This paper will be followed by subsequent papers to describe the research conduct and outcomes.

\section{References}

Ackoff, R. 2004. Transforming the systems movement. Opening Speech at Third International Conference on Systems Thinking in Management (Philadelphia, PA).

Beder, S. 1999. Beyond technicalities: Expanding engineering thinking. Journal of Professional Issues in Engineering Education and Practice 125(1): 12-18.

Bernold, L.,.O'Brien, T. and Akroyd, D. 1998. Meyers-Briggs type indicator and academic achievement in engineering education. International Journal of Engineering Education 14(5): 311-315.

Checkland, P. 1999. Systems thinking, systems practice, soft systems methodology: A 30year retrospective. West Sussex, England: John Wiley and Sons. 
Clup, G. and Smith, A. 2001. Understanding psychological type to improve project team performance. Journal of Management in Engineering 17(1): 24-33.

Cooke, N., et.al. 2004. Advances in Measuring Team Cognition. Team Cognition, ed. E. Salas and S.M. Fiore. Washington, DC: American Psychological Association.

Davidz, H., Nightingale, D., and Rhodes, D. 2005. Enablers and barriers to systems thinking development: Results of a qualitative and quantitative study. In Proceedings of the 3rd Conference on Systems Engineering Research (Hoboken, NJ): CSER.

Davidz, H. 2006. Enabling systems thinking to accelerate the development of senior systems engineers. MIT Doctoral Thesis, Massachusetts Institute of Technology, Cambridge, MA: MIT.

Dong, Q. 1999. Representing Information Flow and Knowledge Management in Product Design Using Design Structure Matrix. Master of Science thesis, Massachusetts Institute of Technology. Cambridge, MA: MIT.

Dorner, D. 1996. The logic of failure: Recognizing and avoiding error in complex situations. New York, NY: Perseus Books Group.

Durling, D. 2003. Horse or cart? Designer creativity and personality, In Proceedings of the 5th European Academy of Design Conference (Barcelona, Spain).

Dym, C., et.al. 2005. Engineering design thinking, teaching, and learning. Journal of Engineering Education 94(1):103-120.

Entin, E., MacMillan, J. and Serfaty, D. 2004. Communication overhead: The hidden cost of team cognition. Team Cognition, ed. E. Salas and S.M. Fiore. Washington, DC: American Psychological Association.

Frank, M. 2000. Engineering systems thinking and systems thinking. Systems Engineering 3(3): 163-168.

Gharajedaghi, J. 1999. Systems thinking: Managing chaos and complexity. Burlington, MA: Butterworth-Heinemann.

Gigerense, G., et.al. 1999. Simple heuristics that make us smarter. New York, NY: Oxford University Press.

Glaser, B. and Strauss, A. 1967. The discovery of grounded theory. Chicago, IL: Aldine Publishing Company.

Hackman, J. R. 2002. Leading teams. Boston, MA: Harvard Business School Press.

Jansma, P. and Jones, R. 2006. Advancing the practice of systems engineering at JPL. In Proceedings of the IEEE Aerospace 2006 (Big Sky, MT): IEEE.

Lamb, C.T., and Rhodes, D.H. 2007. Standardized process as a tool for higher level systems thinking. In Proceedings of the INCOSE International Symposium 2007 (San Diego, CA): INCOSE.

$\square-$ 2007. Promoting systems thinking through alignment of culture and process: Initial results. In Proceedings of the 5th Conference on Systems Engineering Research (Hoboken, NJ): CSER.

Lemos, R. 2006. NASA fights premature graying. Wired News. http://www.wired.com/news/technology/space/0, 71822-0.html.

Lerch, R., Espinosa, J. and Kraut, R. 2004. Explicit versus implicit coordination mechanisms and task dependencies: One size does not fit all. Team Cognition, ed. E. Salas and S.M. Fiore. Washington, DC: American Psychological Association.

Lynn, G., Akgun, A. and Reilly, R. 2005. Multi-dimensionality of learning in new product development teams. Journal of Innovation Management 5(2): 57-72. 
Mohammed, S. and Dumville, B. 2001. Team mental models in team knowledge framework: Expanding theory and management across disciplinary boundaries. Journal of Organizational Behavior 22(1): 89-106.

Pajerek, L. 2000. Processes and Organizations as Systems. Systems Engineering 3(2): 103-111.

Rhodes, D.H. and Hastings, D. 2004. The Case for Evolving Systems Engineering as a Field within Engineering Systems. MIT Engineering Systems Symposium (Cambridge, MA).

Richmond, B. 1993. Systems thinking: Critical thinking skills for the 1990s and beyond. System Dynamics Review 9(2): 113-133.

Saunders, T., et.al. 2005. System-of-systems engineering for Air Force capability development. US Air Force Scientific Advisory Board.

Schein, E., Organizational Culture and Leadership, Jossey-Bass, San Francisco, 2004.

Senge, P. 2006. The fifth discipline. New York, NY: Doubleday.

Stephens, R. 2003. Ensuring aerospace skills for the future: The birth to work pipeline. In Proceedings of the AIAA/ICAS International Air and Space Symposium and Exposition: The Next 100 Years (Dayton, OH). AIAA.

Sterman, J. 2000. Business Dynamics: Systems Thinking and modeling for a complex world. New York, NY: McGraw-Hill.

Strauss, A., and Corbin, J. 1998. Basics of qualitative research. Thousand Oaks, CA: Sage Publications.

Sweeney, L. and Sterman, J. 2000. Bathtub dynamics: Initial results of a systems thinking inventory. System Dynamics Review 16(4): 249-294.

Thompson, G. and Lordan, M. 1999. A Review of creativity principles applied to engineering design. Journal of Process Mechanical Engineering 213(1): 17-31.

Utter, D.A. 2007. Collaborative Distributed Systems Engineering, Master of Science Thesis, Engineering Systems Division. Cambridge, MA: MIT.

Volger, A. 2002. The Munich model: Creating and environment for space architecture development. In Proceedings of the AIAA Space Architecture Symposium (Houston, TX). AIAA.

Waszakm M., et.al. 1998. Modelling and analysis of multidisciplinary research teams at NASA Langley Research Center: A systems thinking approach, In Proceedings of the 7th AIAA/USAF/NASA/ISSMO Symposium on Multidisciplinary Analysis and Optimization (St. Louis, MO). AIAA.

Wesenberg, P. 1989. A multi-disciplinary approach to creativity in organisations, Working paper, Norwegian Center for Leadership Development.

Yin, R. 2003. Case study research design and methods. Thousand Oaks, CA: Sage Publications.

\section{Biography}

Caroline Twomey Lamb is a doctoral candidate at MIT's Lean Advancement Initiative (LAI). She is pursuing her degree through MIT's Department of Aeronautics and Astronautics. Her primary interests focus on enterprise issues within the aerospace industry. Past research and experience includes modeling and analyzing turbine quality control procedures and composites fabrication and structural testing. Ms. Lamb received 
her S.B and S.M from MIT in 2003 and 2005 respectively. She currently serves as the Student Liaison to the American Institute of Aeronautics and Astronautics Board of Directors.

Dr. Donna H. Rhodes is a Senior Lecturer and Principal Research Scientist in the MIT Engineering Systems Division. Previously, Dr. Rhodes held senior management positions in several corporations. She is a co-founder of the MIT Systems Engineering Advancement Research Initiative (SEAri), directing its research program and advising graduate students. She also leads research in enterprise systems engineering for the Lean Advancement Initiative at MIT. She is a Past President, Fellow, and Founder of INCOSE, and director of the SEANET doctoral student network. She has published numerous papers in the field of systems engineering. Her research focuses on architecting and design of complex systems, systems-of-systems, and enterprises. She holds a M.S. and Ph.D. in Systems Science from T.J. Watson School of Engineering at Binghamton University. 